\title{
Intractable itch relieved by 4-phenylbutyrate therapy in patients with progressive familial intrahepatic cholestasis type 1
}

Yasuhiro Hasegawa ${ }^{1 \dagger}$, Hisamitsu Hayashi ${ }^{2^{* \dagger}}$, Sotaro Naoi ${ }^{2}$, Hiroki Kondou ${ }^{1 *}$, Kazuhiko Bessho ${ }^{1}$, Koji Igarashi ${ }^{3}$, Kentaro Hanada ${ }^{4}$, Kie Nakao ${ }^{1}$, Takeshi Kimura ${ }^{1}$, Akiko Konishi ${ }^{1}$, Hironori Nagasaka ${ }^{5}$, Yoko Miyoshi ${ }^{1}$, Keiichi Ozono $^{1}$ and Hiroyuki Kusuhara ${ }^{2}$

\begin{abstract}
Background: Progressive familial intrahepatic cholestasis type 1 (PFIC1), an inherited liver disease caused by mutations in ATP8B1, progresses to severe cholestasis with a sustained intractable itch. Currently, no effective therapy has been established for PFIC1. Decreased function of the bile salt export pump (BSEP) in hepatocytes is suggested to be responsible for the severe cholestasis observed in PFIC1. We found a previously unidentified pharmacological effect of 4-phenylbutyrate (4PB) that increases the expression and function of BSEP. Here, we tested 4PB therapy in three patients with PFIC1.
\end{abstract}

Methods: The therapeutic potency of $4 \mathrm{~PB}$ in these patients was tested by oral administration of this drug with gradually increasing dosage (200, 350, and 500 mg/kg/day) for 6 months. Biochemical, histological, and clinical data were collected.

Results: 4PB therapy had no beneficial effect on the patients' liver functions, as assessed by biochemical and histological analyses, despite an increase in hepatic BSEP expression. However, therapy with 4PB at a dosage of 350 or $500 \mathrm{mg} / \mathrm{kg} /$ day significantly relieved the intractable itch. Serum levels of potential pruritogens in cholestasis were much higher than the reference ranges during the $4 \mathrm{~PB}$ therapy.

Conclusions: $4 \mathrm{~PB}$ therapy may be a new medication for patients with intractable cholestatic pruritus and may improve quality of life for patients and their families.

Keywords: Pediatric liver disease, Cholestasis, PFIC1, Pruritus, 4PB

\section{Background}

Progressive familial intrahepatic cholestasis type 1 (PFIC1), a rare inherited autosomal recessive liver disease caused by mutations in ATP8B1, is characterized primarily by normal serum gamma-glutamyl transferase (GGT), intrahepatic cholestasis and jaundice in the first year of life [1]. This disease progresses to severe cholestasis with sustained intractable itching, jaundice, watery diarrhea, failure to thrive,

\footnotetext{
* Correspondence: hayapi@mol.f.u-tokyo.ac.jp; kondou@ped.med.osaka-u.ac.jp ${ }^{\dagger}$ Equal contributors

2Laboratory of Molecular Pharmacokinetics, Graduate School of

Pharmaceutical Sciences, The University of Tokyo, 7-3-1 Hongo, Bunkyo-ku,

Tokyo 113-0033, Japan

'Department of Pediatrics, Osaka University Graduate School of Medicine, 2-2

Yamada-oka, Suita, Osaka 565-0871, Japan

Full list of author information is available at the end of the article
}

pancreatitis, and deafness, resulting in liver failure and death before adulthood [2]. The main complaint in the clinical course of patients with PFIC1 is often intractable itching, which significantly disrupts the patients' ability to sleep and thus decreases the quality of life of patients and their families [3]. No effective medical therapy for this disease is currently available [3]. Even liver transplantation is insufficient to improve the clinical course and outcomes of patients with PFIC1 because of steatosis and fibrosis [4].

ATP8B1 is a member of the P4 subfamily of P-type adenosine triphosphatases and is expressed on the apical membrane of many epithelial cells including hepatocytes. ATP8B1 translocates phosphatidylserine (PS) from the outer leaflet to the inner leaflet and thereby contributes to 
making the hepatocanalicular membrane $(\mathrm{CM})$ a rigid, liquid-ordered membrane [5,6]. In patients with PFIC1, the well-organized aminophospholipid asymmetry of the $\mathrm{CM}$ is disrupted by the impaired function of ATP8B1, leading to a decrease in the transport activity of the bile salt export pump (BSEP), an ABC transporter that is localized on the $\mathrm{CM}$ and that predominantly mediates biliary excretion of bile salts [7-10], and subsequently to the onset of severe intrahepatic cholestasis [11]. Alternatively, in patients with PFIC1, nuclear translocation of the farnesoid $\mathrm{X}$ receptor (FXR), a transcription factor that controls bile acid homeostasis, is disrupted and causes a decrease in BSEP expression at the CM because of mass action related to the decreased expression of BSEP mRNA [12]. Therefore, in either cause of PFIC1, an increase in BSEP function is expected to compensate for the reduced capacity for bile salt excretion into bile in patients with PFIC1, and may improve their liver function.

We have published experimental evidence that 4phenylbutyrate (4PB), a drug used to treat ornithine transcarbamylase deficiency (OTCD), has another newly identified pharmacological effect that increases the hepatocanalicular expression of BSEP and the hepatocyte capacity for biliary excretion of bile salts when given at a clinically relevant concentration in OTCD patients [13]. The higher BSEP expression level in liver specimens from patients with OTCD after 4PB therapy compared with that before 4PB therapy suggests that $4 \mathrm{~PB}$ treatment increases BSEP expression in humans [14]. Furthermore, our group and Gonzales et al. reported recently that $4 \mathrm{~PB}$ therapy restored decreased BSEP expression, improved liver functions in histological and biochemical analysis, and relieved intractable pruritus in patients with PFIC type 2 (PFIC2), an inherited autosomal recessive liver disease caused by mutations in BSEP [15,16]. PFIC2 patients present with similar clinical symptoms and biological parameters as PFIC1 patients $[17,18]$. Together, these results suggest the possibility that $4 \mathrm{~PB}$ may be a potential therapeutic compound for PFIC1 patients that could act to restore the reduced capacity of biliary excretion of bile salt through increasing BSEP expression on the CM.

To test this hypothesis, our current study investigated the effects of 4PB therapy in three PFIC1 patients. 4PB was administered orally with gradually increasing dosage (200, 350, and $500 \mathrm{mg} / \mathrm{kg} /$ day) for 6 months. We collected data on serum liver tests, histological analyses, pruritus score, and the clinical course for these patients.

\section{Methods}

We obtained approval for the study from the institutional ethics review boards. Informed consent was obtained from the patients' parents before assessment because the patients were younger than 18 years of age.
A detailed description of the materials and methods is presented in the Additional file 1. All materials and methods used standard techniques and commercially available reagents.

\section{Patients}

The patients enrolled in our study were three Japanese boys (Patient 1, 2, and 3) who were seen at Osaka University Hospital in 2012 and aged 2-, 6-, and 16-years old, respectively. All three patients developed hepatocellular cholestasis with mild elevation of serum aspartate aminotransaminase (AST) and alanine amino transaminase (ALT) levels and normal GGT levels as infants and experienced sleep disturbance because of intractable itch at around 4, 6, and 4 months of age, respectively. Patient 3 had difficulty in getting to sleep by the end of elementary school. Despite treatment with drugs including ursodeoxycholic acid, topical steroids, and antihistamine agents for $1.5,5$, and 15 years in patient 1,2 , and 3 , respectively, these patients continued to experience severe cholestasis with sustained intractable itch, jaundice, diarrhea, and failure to thrive, which are typical clinical symptoms of PFIC1. The patients and/or their families preferred medical treatment to surgical procedure like partial external biliary diversion. Therefore, the patients were enrolled in this clinical study. The administration of original drugs was maintained during and after the course of $4 \mathrm{~PB}$ treatment. The drugs given to the patients before, during, and after the course of this study are listed in the Additional file 1: Table S1.

\section{Sequence analysis of $A T P 8 B 1$ and $A B C B 11$}

Genomic DNA was isolated from peripheral blood leukocytes using a Wizard Genomic DNA Purification Kit (Promega, Madison, WI), and all exons of ATP8B1 and $A B C B 11$ and flanking intron-exon boundaries were analyzed as described previously $[16,19,20]$.

\section{Treatment of PFIC1 patients with 4PB}

Oral administration of 4PB (Ammonaps; Swedish Orphan Inter AB., Stockholm, Sweden) was started at a daily dosage of $200 \mathrm{mg} / \mathrm{kg} /$ day divided into four doses a day. After 1 month, the dosage was increased to $350 \mathrm{mg} / \mathrm{kg} /$ day and this was maintained for an additional month. Because neither a therapeutic effect nor any side effects were observed, the dosage was increased to $500 \mathrm{mg} / \mathrm{kg} /$ day, which is the clinically relevant dosage for OTCD, and this dosage was maintained for the next 4 months. A liver biopsy sample was collected 1 day before and after the course of 4PB treatment. A part of the sample was preserved in RNAlater (Qiagen, Hilden, Germany) for RNA preparation and stored at $-20^{\circ} \mathrm{C}$. Another portion was fixed in $10 \%$ formaldehyde at room temperature for 
histological analysis, and the remaining portion was snapfrozen in liquid nitrogen for preparation of membrane fractions and stored at $-70^{\circ} \mathrm{C}$ in a deep freezer. Serum was collected before, during, and after the course of 4PB treatment. Liver function tests were performed using standard methods immediately after collection, and the remaining specimens were preserved at $-70^{\circ} \mathrm{C}$ for further analysis.

\section{Pruritus evaluation}

Pruritus severity was scored as reported previously [21]: 0 , none; 1 , mild scratching when undistracted; 2, active scratching without abrasion; 3, abrasions; or 4, cutaneous mutilation, with bleeding and scarring.

\section{Quantitative determination of pruritogen levels in serum}

The concentration and activity of autotaxin (ATX) in serum were assessed using a specific two-site enzyme immunoassay and the measurement of choline liberated from the substrate lysophosphatidylcholine as described previously $[16,22]$.

\section{Histological analysis of the patients' liver specimens}

Liver biopsy specimens were fixed in $10 \%$ formalin and embedded in paraffin. Four-micrometer-thick sections were prepared from the liver specimens and subjected to hematoxylin-eosin (HE) staining and immunohistochemistry followed by microscopic analysis with an Olympus CX41 or Olympus BX40 microscope (Olympus, Tokyo, Japan) to evaluate the degree of cholestasis, fibrosis, and inflammation in the liver tissues.

\section{Preparation of crude membrane, nuclear, and cytosolic} fractions from the patients' liver specimens

Liver specimens from the patients were homogenized in hypotonic buffer (1 mM EDTA, $5 \mathrm{mM}$ sodium phosphate, $\mathrm{pH}$ 7.0) supplemented with protease inhibitor cocktails (Sigma-Aldrich, St. Louis, MO) using a QIAshredder (Qiagen), and then centrifuged at $800 \times \mathrm{g}$ for $10 \mathrm{~min}$ at $4^{\circ} \mathrm{C}$. The supernatant was ultracentrifuged at $100,000 \times \mathrm{g}$ for $1 \mathrm{~h}$ at $4^{\circ} \mathrm{C}$ and the pellet and supernatant were used as the crude membrane and cytosolic fractions, respectively. After centrifugation at $800 \times \mathrm{g}$, the pellet was suspended with high salt buffer (20 mM Tris- $\mathrm{HCl}$ pH 7.9, $400 \mathrm{mM}$ $\mathrm{NaCl}, 0.1 \mathrm{mM}$ EDTA, $0.1 \mathrm{mM}$ EGTA Na, 0.1\% NP-40, 1 mM DTT, $10 \%$ glycerol, $0.1 \%$ protease inhibitor cocktail), incubated on ice for $50 \mathrm{~min}$ with vortex mixing every $10 \mathrm{~min}$, and centrifuged at $3000 \times \mathrm{g}$ for $10 \mathrm{~min}$ at $4^{\circ} \mathrm{C}$, and the supernatant was used as the nuclear extract.

\section{Immunoblotting}

Specimens were loaded into each well of a 7\% SDSPAGE plate with a $3.75 \%$ stacking gel, and subjected to immunoblotting as described previously [13,14,23]. Immunoreactivity was detected with an ECL Advance ${ }^{\mathrm{TM}}$
Western Blotting Detection Kit (Amersham Biosciences, Piscataway, NJ). The intensity of the band was quantified by MultiGauge software (version 2.0; Fujifilm, Tokyo, Japan). Expression levels of ATP8B1, BSEP, and CDC50A were normalized by the expression of $\mathrm{Na}^{+}, \mathrm{K}^{+}$-ATPase $\alpha 1$ subunit $(\mathrm{NaK} \alpha 1)$, which was not affected by the treatment with 4PB (data not shown).

\section{Results}

\section{Diagnosis of PFIC1 in the patients}

Sequencing analysis of all encoding exons and flanking intron-exon boundaries of ATP8B1 identified a heterozygous mutation c.3033-34del (frame shift or splicing defect) in patient 1 and a heterozygous mutation c.158789del (p.F529del) in patient 2 (Table 1). The c.1587-89del (p.F529del) mutation has been reported previously in European PFIC1 patients of Caucasian descent [19]. Although no other mutations were found in ATP8B1 on the other allele, as was the case for several PFIC1 patients reported in a previous study [19], both patients were diagnosed with PFIC1 because they exhibited the typical clinical symptoms of PFIC1 and because of the low mRNA expression and no detectable protein expression of ATP8B1 in their liver biopsy specimens (Figure 1A, $B)$. The near absence and marked decrease of hepatic ATP8B1 mRNA in patients 1 and 2 may be explained by other mutations in the promoter region and/or untranslated region (UTR) of $A T P 8 B 1$ on the other allele that affect transcription of $A T P 8 B 1$ and/or stabilization of ATP8B1 mRNA. Patient 3 was diagnosed with PFIC1 because of lower mRNA and protein expression of ATP8B1 in his liver specimen (Figure 1A, B) and because of his clinical symptoms including intrahepatic cholestasis with normal GGT, intractable itching, failure to thrive, and deafness. The sequencing analysis of $A T P 8 B 1$ in patient 3 identified c.234C $>$ G (p.H78Q) (rs3745079) and c.2021 T > C (p.M674T) (rs35470719) mutations on one allele and a mutation c.1729A > G (p.I577V) (rs3745078) on the other allele. However, given that these mutations had no significant effect on mRNA and protein expression, trafficking to the plasma membrane, and PS flippase activity of ATP8B1 in in vitro studies (Additional file 2: Figure S1 A-D), it is likely that the decreased mRNA and protein expression of ATP8B1 in this patient is caused by mutations in the promoter region and/or UTR of $A T P 8 B 1$ that affect transcription of $A T P 8 B 1$ and stabilization of ATP8B1 mRNA, but not by the mutations analyzed in this study.

\section{Therapeutic effect of 4PB in PFIC1 patients}

During the period of $4 \mathrm{~PB}$ treatment, no improvement was observed in liver function tests for any of the patients (Figure 2). However, their itching started to attenuate in patient 1 three weeks after the dosage of $4 \mathrm{~PB}$ 
Table 1 ATP8B1 mutation and biochemical parameters in patients enrolled in this study

\begin{tabular}{|c|c|c|c|c|c|c|c|}
\hline & Sex & Age & $A T P 8 B 1$ mutation in allele 1 & $A T P 8 B 1$ mutation in allele 2 & AST/ALT/GGT (U/L) & $\begin{array}{c}\text { T-Bil/D-Bil/BA } \\
(\mu \mathrm{M})\end{array}$ & $\begin{array}{c}\text { Pruritus } \\
\text { score }\end{array}$ \\
\hline Patient 1 & M & 2 & c. $3033-3034 d e l$ (Frame shift) & Not found & $83 / 60 / 36$ & $4.8 / 4.6 / 301.3$ & 4 \\
\hline Patient 2 & M & 6 & c.1585-1587TTCdel (p.F529del) & Not found & $81 / 15 / 27$ & $3.4 / 3.2 / 214.1$ & 4 \\
\hline Patient 3 & M & 16 & c.234C > G (p.H78Q) c.202IT>C (p.M674T) & c. $1729 \mathrm{~A}>\mathrm{G}(\mathrm{p} .1577 \mathrm{~V})$ & $63 / 51 / 19$ & $0.6 / 0.3 / 54.6$ & 4 \\
\hline
\end{tabular}

was increased to $350 \mathrm{mg} / \mathrm{kg} /$ day, in patient 2 one week after the dosage was increased to $500 \mathrm{mg} / \mathrm{kg} /$ day, and in patient 3 four weeks after the dosage of $4 \mathrm{~PB}$ was increased to $350 \mathrm{mg} / \mathrm{kg} /$ day. The itching score declined from 4 to 2 in all patients (Figure 3A, B). Although there were multiple dark erosions in the skin of all patients and elephantiasis in patient 3 due to intense and continual scratching, after the onset of $4 \mathrm{~PB}$ treatment at the dosage of 350 or $500 \mathrm{mg} / \mathrm{kg} / \mathrm{day}$, the frequency and intensity of skin scratching was markedly decreased, leading to diminished skin erosion and hemorrhage and improved skin appearance (Figure 3C). At the end of the therapy, hemorrhage and eschar on the patients' skin were diminished and areas of fresh normal skin were evident. The parents of the patients noted an improvement in sleep disturbance during the night and in their child's skin condition. In contrast to the relief of the itching, the serum levels of bile acids and ATX and of ATX activity, all of which have been proposed as potential pruritogens in cholestasis [24], were not decreased by $4 \mathrm{~PB}$ therapy in any of the patients (Figures $2 \mathrm{~B}, 3 \mathrm{~A}, \mathrm{~B}$ ). The itch remained unchanged for 6,4 , and 6 weeks after cessation of $4 \mathrm{~PB}$ therapy in patients 1,2 , and 3 , respectively, but then gradually exacerbated, resulting in

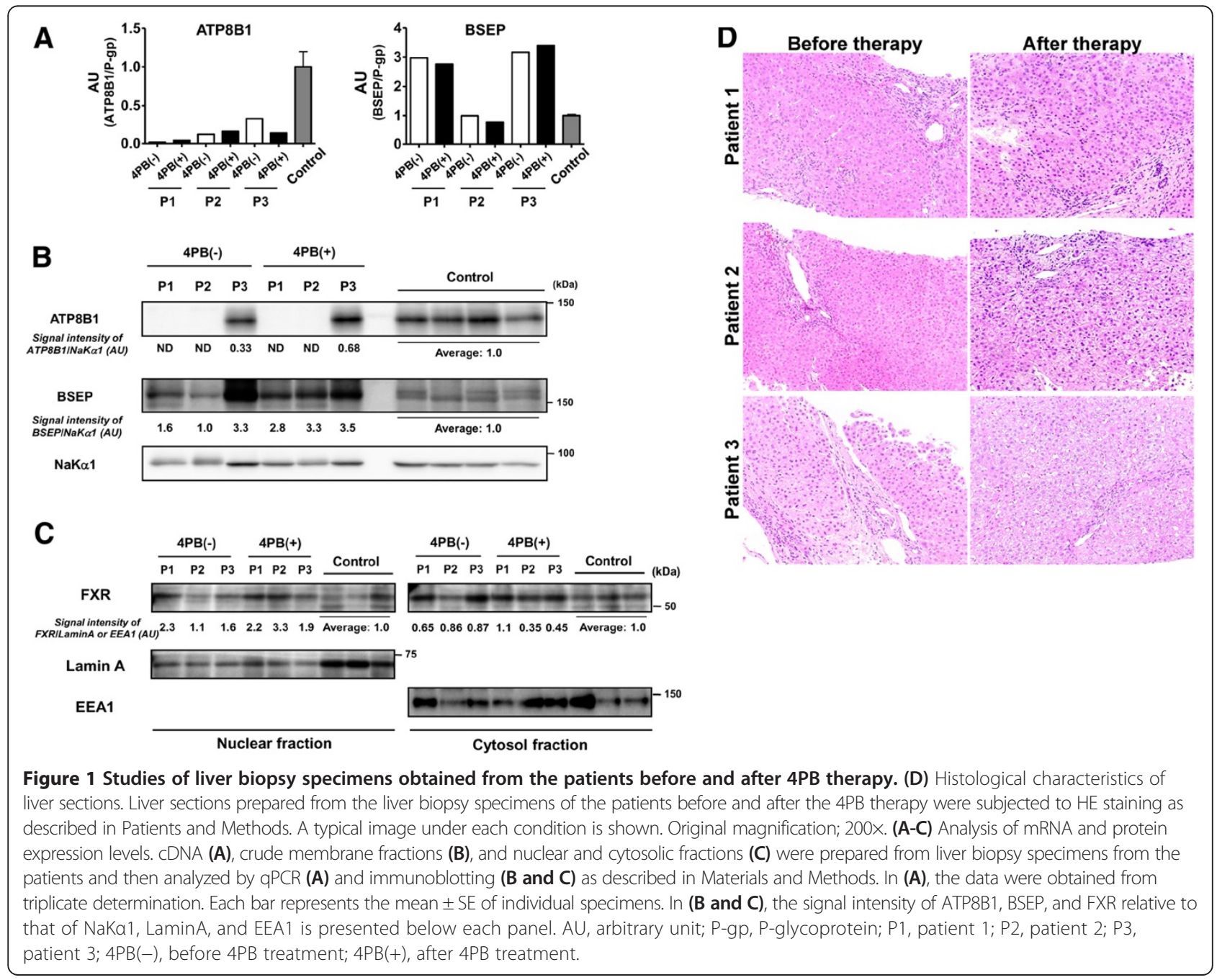




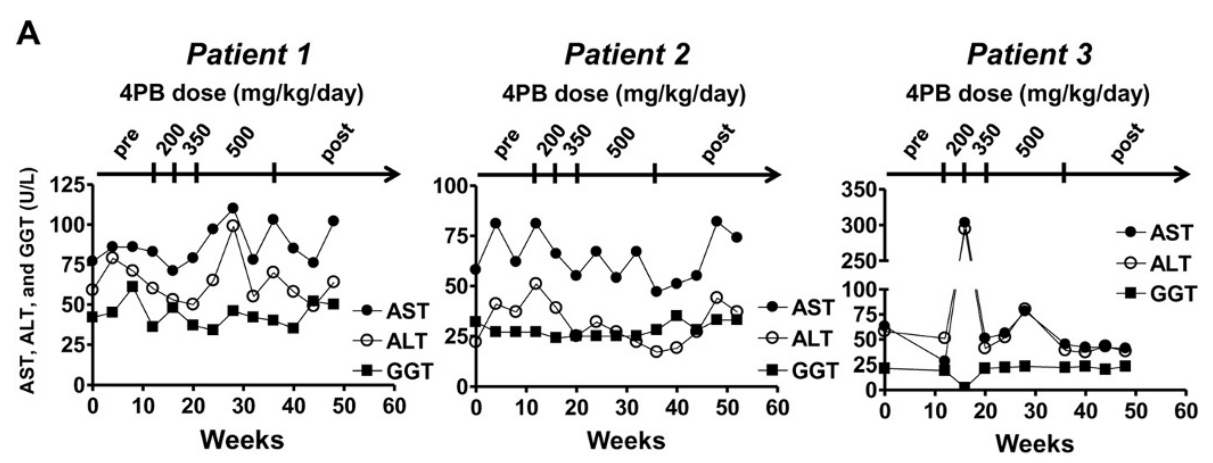

B
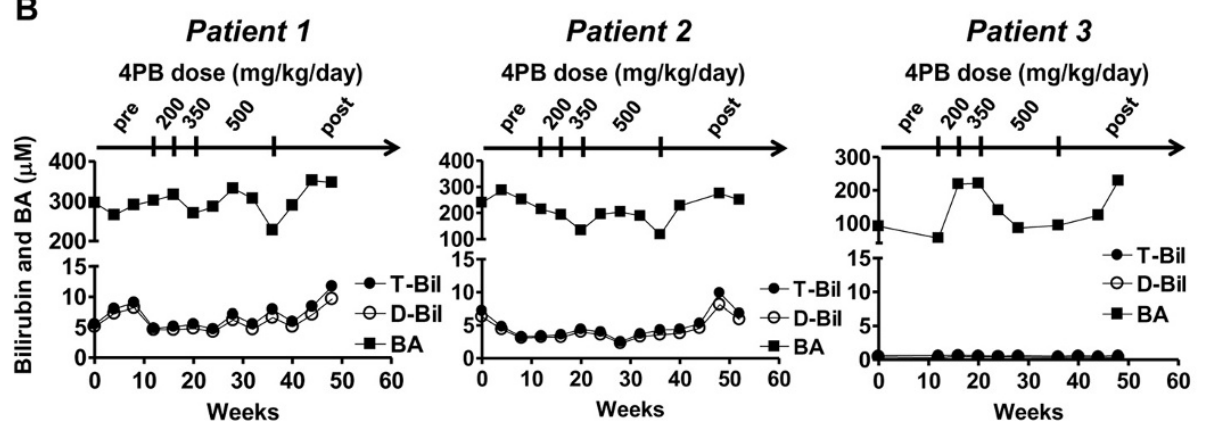

Figure 2 Liver function testing before, during and after the course of 4PB therapy in the patients. Serum AST, ALT, GGT (A), total bilirubin, direct bilirubin, and bile acids (B) levels were monitored before, during and after the 4PB therapy. T-Bil, total bilirubin; D-Bil, direct bilirubin; BA, bile acids.

regeneration of erosion and hemorrhage again because of intense scratching. In all the patients, 8 weeks after the end of $4 \mathrm{~PB}$ therapy, the itching score returned to values equal to those before the treatment (Figure 3A, B). Because of the bad taste of $4 \mathrm{~PB}$, all patients had difficulty taking the doses at the beginning of the therapy. However, their parents noted the improvement in their child's sleep during the night and in the skin conditions, and encouraged their child to continue the therapy. No patients dropped out of this study. No severe side effects were observed during and after the 4PB therapy. The temporary elevation of AST and ALT concentrations after patient 3 began $4 \mathrm{~PB}$ treatment at $200 \mathrm{mg} / \mathrm{kg} /$ day was thought to be caused by adenovirus infection and not by any adverse effect of the $4 \mathrm{~PB}$ therapy, because both markers increased promptly after the adenovirus infection appeared and decreased to the basal levels concurrently with recovery from the infection (Figure 2A).

\section{Effect of 4PB therapy on liver histology and BSEP expression in PFIC1 patients}

A liver biopsy was performed 6 months after the initiation of 4PB therapy and compared with the specimens obtained 1 day before onset of 4PB therapy. The specimens of the age-matched control subjects were obtained from OTCD patients without administration of $4 \mathrm{~PB}$ when they underwent liver transplantation. qPCR and immunoblot analysis demonstrated that even in the specimens taken after the 4PB therapy, the mRNA expression of ATP8B1 was still much lower in all the patients than that of age-matched control subjects, and ATP8B1 protein expression in the membrane fraction was undetectable in patients 1 and 2 . In patient 3 , ATP8B1 expression was increased about 2-fold by 4PB therapy, but still lower than that in age-matched control subjects (Figure 1B, C). This result was consistent with the lack of change in ATP8B1-FLAG ${ }^{\text {F529del }}$ and a 2.1- and 1.3-fold increase in ATP8B1 $1^{\mathrm{H} 78 \mathrm{Q}+\mathrm{M} 674 \mathrm{~T}}$-FLAG and ATP8B1 ${ }^{1577 V}$-FLAG in UPS-1 cells after treatment with $4 \mathrm{~PB}$ at a clinically relevant concentration $(1 \mathrm{mM})$ (Additional file 3: Figure S2). BSEP protein expression was increased after $4 \mathrm{~PB}$ therapy without affecting its mRNA expression as is the case in patients with PFIC2 and OTCD (Figure 1A, B) $[14,16]$. The amount of FXR in the nuclear fraction of cells from the PFIC1 patients was nearly equal to or a little higher than that of age-matched control subjects, and was not significantly affected by $4 \mathrm{~PB}$ therapy except for patient 2 (Figure 1C). In this patient, the amount of FXR was increased 3-fold after 4PB therapy. Histological analysis showed that in the specimens obtained from the PFIC1 patients before this study, the portal area was enlarged and had progressed to fibrosis with mild inflammation and partly bridging fibrosis and 

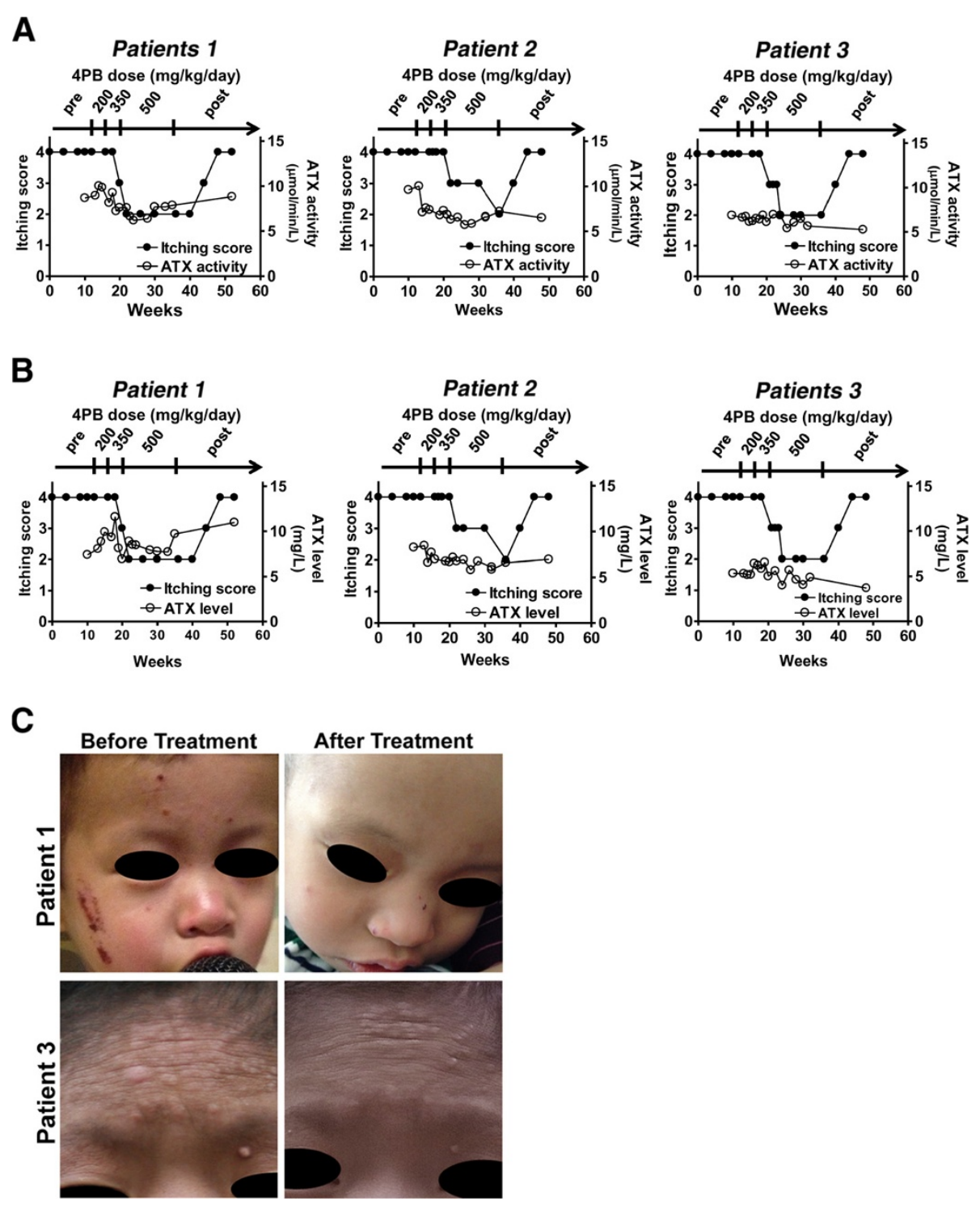

Figure 3 Itching intensity in the patients before, during, and after the course of 4PB therapy. (A, B) Correlation diagram of itching scores for the PFIC1 patients with serum ATX activity (A) and ATX level (B) in the patients before, during, and after 4PB therapy. Pruritus severity was scored ranging from 0 (no pruritus) to 4 (cutaneous mutilation, with bleeding and scarring) as described in Patients and Methods. (C) Skin of the patients before and after the 4 PB therapy.

that $4 \mathrm{~PB}$ therapy caused no remarkable change in these features (Figure 1D).

\section{Discussion}

The main complaint in the clinical course of PFIC1 is often the intractable itching, which significantly disrupts the patients' activities of daily living, work productivity, and ability to sleep, and thereby decreases the quality of life for them and their families [2]. Topical steroids, antihistamine agents, and rifampicin are the only prescribed drugs currently available for the cholestatic pruritus in PFIC1 patients, but these medications are often ineffective, as was the case for the patients enrolled in this study
[3]. The principal finding of our current study is that $4 \mathrm{~PB}$ therapy at a clinically relevant dosage used in OTCD patients markedly relieved intractable cholestatic pruritus in PFIC1 patients. At the end of the therapy, hemorrhage and eschar on the patients' skin were diminished, and areas of fresh normal skin appeared as the frequency and intensity of skin scratching decreased. Parents of the patients noted that 4PB therapy made it easier for their child to get to sleep and markedly reduced their child's sleep disturbance during the night. Thus, favorable outcomes of 4PB therapy were observed, and these were dose-dependent, although the patients and their families in this clinical study were not 
informed of the detailed protocol. The facts that the patients and their families were not informed of this aspect and that the drugs prescribed for $1.5,5$, and 15 years in patient 1,2 , and 3 , respectively, before this study were ineffective in improving the sustained intractable itch suggest that the relief of refractory cholestatic itching in these PFIC1 patients occurred because of the 4PB therapy and not because of a placebo effect or an effect of other medications.

The visual analog scale (VAS) is the most general method for assessing pruritus, but is not easily applied to younger children because VAS is a graphic method based on the patients' subjective rating of symptoms [25]. Therefore, in this study, the patients' itching was evaluated on the basis of cutaneous findings and scored according to the method used in a previous report [21]. A potential concern when using this method is that the improvement in itch may be underestimated. The severity of skin erosion, thickness, and cicatrices depends on the duration of the itch and can vary in the patients scored as 4 . Therefore, there may have been a time lag in the improvements in skin appearance even if the frequency and intensity of scratching decreased to the same degree in all patients. In this study, the improvement of pruritus in patient 3 , who experienced intractable pruritus for a longer period than patients 1 and 2, might have been underestimated compared with that in patients 1 and 2. To overcome this limitation, it might be better to apply the 5-D itch scale, a recently developed method that assesses the subjective symptoms of itching in patients from five dimensions: degree, duration, direction, disability and distribution [25]. In the 5-D itch scale, but not in the VAS, the subjective symptoms reported by the patient can be supported by his/her family. Therefore, in future clinical studies, the change in chronic pruritus in younger patients should be explored using the 5-D itch scale as well as the method used in this study. This should allow a more accurate determination of the clinical outcomes and the beneficial effects of 4PB therapy on cholestatic pruritus in PFIC1 patients.

In contrast to the relief of intractable cholestatic itching, beneficial effects of 4PB therapy were not observed in liver function tests and liver histology despite an increase in BSEP expression in liver membrane fractions as reported previously (Figure 1B, D and 2) $[14,16]$. This could be because a two- to three-fold increase in BSEP expression may be insufficient to improve intrahepatic cholestasis in PFIC1 patients. The other possible reason is that the transport activity of BSEP is lost completely in PFIC1 patients because of the disrupted lipid asymmetry of the CM $[6,11]$ and, consequently, the increase in BSEP expression by 4PB therapy cannot compensate for the reduced capacity of bile salt excretion into bile. Although no remarkable improvement in liver function was observed in the patients in our study, 4PB therapy might have therapeutic potency for specific PFIC1 patients with mutations in ATP8B1 that attenuate ATP8B1 expression, but do not affect its protein activity. An in vitro analysis has shown that treatment with $4 \mathrm{~PB}$ partially restored the decreased expression of ATP8B1 caused by p.G308V, p.D454G, and p.D544N, all of which are naturally occurring mutations [26]. Future clinical studies should validate the therapeutic effect of $4 \mathrm{~PB}$ and its safety for use in PFIC1 patients who carry mutations that attenuate ATP8B1 expression but do not affect its protein activity.

At present, the mechanism underlying the relief of cholestatic pruritus by 4 PB therapy remains to be elucidated. No decrease in the factors suspected to be causally associated with cholestatic pruritus (Figures 2, 3A, B) are consistent with the observation in PFIC2 patient during 4PB therapy [16]. 4PB and/or its metabolites may modulate the local concentrations of these pruritogens, which may not have been detected by systemic measurements. The physiological function of ATX, an enzyme secreted extracellularly that generates lysophosphatidic acid, is thought to be mediated predominantly by activation of G protein-coupled receptors (GPCRs) [27]. TGR5, a GPCR activated by bile salts, in sensory nerves could contribute to bile salt-induced itching [28]; if so, $4 \mathrm{~PB}$ and/or its metabolites might antagonize the GPCRs responsible for itch signaling and therefore attenuate the activation of sensory neurons. Alternatively, 4PB therapy may disrupt pruriceptive projections to the brain through distribution of $4 \mathrm{~PB}$ and/or its metabolites into the brain [29] or may affect pruritogens or antipruritogens that have not been identified yet. Further studies to test these possibilities will provide a better understanding of the mechanisms responsible for the effects of 4PB therapy on cholestatic pruritus and thereby the molecular mechanism of cholestatic pruritus itself. Information obtained from these studies will contribute to the development of new molecular target drugs for cholestatic pruritus, which will hopefully be more effective than $4 \mathrm{~PB}$ and consequently, improve the clinical application of 4PB.

\section{Conclusions}

Our study has provided clinical evidence that 4PB therapy can relieve the refractory itching in PFIC1 patients, and thereby improved the quality of life of the patients and their families. Future clinical studies with more patients and longer time periods than were possible in this study should be undertaken to confirm the favorable effects of 4PB therapy using the 5-D itch scale and the other method used in this study. If confirmed, $4 \mathrm{~PB}$ therapy could become the preferred choice, instead of topical steroids, antihistamine agents, and surgical procedures, 
for attenuating cholestatic pruritus in patients with PFIC1 and benign intrahepatic cholestasis type 1 (BRIC1), a hereditary disorder characterized by mutations in ATP8B1 and by recurrent and intermittent episodes of cholestasis with refractory cholestatic pruritus [30]. 4PB therapy might also be effective for intractable pruritus caused by other cholestatic disorders such as Alagille syndrome. Clinical trials will be required to determine the utility and safety of $4 \mathrm{~PB}$ as a therapy for these diseases.

\section{Additional files}

Additional file 1: Supplemental information.

Additional file 2: Effects of mutations in ATP8B1 on mRNA and protein expression levels, cellular localization, and function of ATP8B1. Additional file 3: Effect of 4PB on the expression levels of ATP8B1 mutants.

\section{Abbreviations}

ALT: Alanine amino transaminase; AST: Aspartate aminotransaminase; ATX: Autotaxin; AU: Arbitrary units; BA: Bile acids; BRIC: Benign intrahepatic cholestasis; BSEP: Bile salt export pump; CM: Canalicular membrane; D-Bil: Direct bilirubin; ER: Endoplasmic reticulum; EV: Empty vector; FITC: Fluorescein isothiocyanate; FXR: Farnesoid X receptor; GGT: Gamma-glutamyl transferase; GPCR: G protein-coupled receptor; HA: Hemagglutinin antigen; HE: Hematoxylin-eosin; $\mathrm{Na}^{+}$: $\mathrm{K}^{+}$-ATPase a1 subunit, NaKa1; ND: Not detected because of low expression; OTCD: Ornithine transcarbamylase deficiency; PFIC: Progressive familial intrahepatic cholestasis; PS: Phosphatidylserine; qPCR: Quantitative polymerase chain reaction; SE: Standard error; T-Bil: Total bilirubin; VAS: Visual analog scale; WT: Wild type; 4PB: 4-phenylbutyrate.

\section{Competing interests}

H.H. has applied a patent on the effect of 4-phenylbutyrate on bile salt export pump (US serial No.13/299,989).

\section{Author contribution}

YH recruited and enrolled the patients, collected specimens from the patients, performed the clinical assessment and follow-up, contributed to data interpretation, and drafted the manuscript together with $\mathrm{HH}$. $\mathrm{HH}$ directed and supervised all of the research and took a lead role in writing the manuscript. SN performed most of the in vitro experiments, contributed to data interpretation, and drafted the manuscript together with $\mathrm{HH}$. $\mathrm{HK}$ and $\mathrm{KB}$ recruited and enrolled the patients, collected specimens from the patients, carried out clinical assessment and follow-up, contributed to data interpretation, and revised the manuscript for intellectual content. KI measured ATX concentration. KH provided UPS-1 cells. KN, TK, and AK collected specimens from the patients and carried out clinical assessment and follow-up. HN helped to make a diagnosis of the patients, and contributed to data interpretation. YM, KO, and HK contributed to data interpretation and revised the manuscript for intellectual content. All authors approved the manuscript before submission.

\section{Acknowledgments}

We thank Dr. Larissa Kogleck (The University of Tokyo, Tokyo, Japan), Dr. Masayoshi Kage, Dr. Ken Tanikawa (Kurume University, Fukuoka, Japan), and Dr. Ayano Inui (Saiseikai Yokohamashi Tobu Hospital, Kanagawa, Japan) for advice on the manuscript. This study was supported by a Grant-in-Aid for Young Scientists (B) (21790146) and Astellas Foundation for Research on Metabolic Disorders to H.H.

\section{Author details}

${ }^{1}$ Department of Pediatrics, Osaka University Graduate School of Medicine, 2-2 Yamada-oka, Suita, Osaka 565-0871, Japan. ${ }^{2}$ Laboratory of Molecular Pharmacokinetics, Graduate School of Pharmaceutical Sciences, The University of Tokyo, 7-3-1 Hongo, Bunkyo-ku, Tokyo 113-0033, Japan. ${ }^{3}$ Bioscience Division,
Reagent Development Department, TOSOH Corporation, 2743-1 Hayakawa, Ayase-shi, Kanagawa 252-1123, Japan. ${ }^{4}$ Department of Biochemistry and Cell Biology, National Institute of Infectious Diseases, 1-23-1, Toyama, Shinjuku-ku, Tokyo 162-8640, Japan. ${ }^{5}$ Department of Pediatrics, Takarazuka City Hospital, 4-5-1 Kohama, Takarazuka-shi, Hyogo 665-0827, Japan.

Received: 31 March 2014 Accepted: 13 June 2014 Published: 15 July 2014

\section{References}

1. Bull LN, van Eijk MJ, Pawlikowska L, DeYoung JA, Juijn JA, Liao M, Klomp LW, Lomri N, Berger R, Scharschmidt BF, Knisely AS, Houwen RH, Freimer NB: A gene encoding a P-type ATPase mutated in two forms of hereditary cholestasis. Nat Genet 1998, 18(3):219-224.

2. Morotti RA, Suchy FJ, Magid MS: Progressive familial intrahepatic cholestasis (PFIC) type 1, 2, and 3: a review of the liver pathology findings. Semin Liver Dis 2011, 31(1):3-10.

3. Suchy FJ, Shneider BL: 14. familial hepatocellular cholestasis. In Liver Disease in Children 3rd Edition. 2007:310-314.

4. Hori T, Egawa H, Takada Y, Ueda M, Oike F, Ogura Y, Sakamoto S, Kasahara M, Ogawa K, Miyagawa-Hayashino A, Yonekawa Y, Yorifuji T, Watanabe K Doi H, Nguyen JH, Chen F, Baine AM, Gardner LB, Uemoto S: Progressive familial intrahepatic cholestasis: a single-center experience of living-donor liver transplantation during two decades in Japan. Clin Transplant 2011, 25(5):776-785.

5. Paulusma CC, Folmer DE, Ho-Mok KS, de Waart DR, Hilarius PM, Verhoeven AJ, Oude Elferink RP: ATP8B1 requires an accessory protein for endoplasmic reticulum exit and plasma membrane lipid flippase activity. Hepatology 2008, 47(1):268-278.

6. Paulusma CC, Groen A, Kunne C, Ho-Mok KS, Spijkerboer AL, Rudi de Waart D, Hoek FJ, Vreeling H, Hoeben KA, van Marle J, Pawlikowska L, Bull LN, Hofmann AF, Knisely AS, Oude Elferink RP: Atp8b1 deficiency in mice reduces resistance of the canalicular membrane to hydrophobic bile salts and impairs bile salt transport. Hepatology 2006, 44(1):195-204.

7. Byrne JA, Strautnieks SS, Mieli-Vergani G, Higgins CF, Linton KJ, Thompson RJ: The human bile salt export pump: characterization of substrate specificity and identification of inhibitors. Gastroenterology 2002, 123(5):1649-1658.

8. Gerloff T, Stieger B, Hagenbuch B, Madon J, Landmann L, Roth J, Hofmann AF, Meier PJ: The sister of P-glycoprotein represents the canalicular bile salt export pump of mammalian liver. J Biol Chem 1998, 273(16):10046-10050

9. Hayashi H, Takada T, Suzuki H, Onuki R, Hofmann AF, Sugiyama Y: Transport by vesicles of glycine- and taurine-conjugated bile salts and taurolithocholate 3-sulfate: a comparison of human BSEP with rat Bsep. Biochim Biophys Acta 2005, 1738(1-3):54-62.

10. Noe J, Stieger B, Meier PJ: Functional expression of the canalicular bile salt export pump of human liver. Gastroenterology 2002, 123(5):1659-1666.

11. Paulusma CC, de Waart DR, Kunne C, Mok KS, Elferink RP: Activity of the bile salt export pump (ABCB11) is critically dependent on canalicular membrane cholesterol content. J Biol Chem 2009, 284(15):9947-9954.

12. Chen F, Ananthanarayanan M, Emre S, Neimark E, Bull LN, Knisely AS, Strautnieks SS, Thompson RJ, Magid MS, Gordon R, Balasubramanian N, Suchy FJ, Shneider BL: Progressive familial intrahepatic cholestasis, type 1, is associated with decreased farnesoid $\mathrm{X}$ receptor activity. Gastroenterology 2004, 126(3):756-764.

13. Hayashi H, Sugiyama Y: 4-phenylbutyrate enhances the cell surface expression and the transport capacity of wild-type and mutated bile salt export pumps. Hepatology 2007, 45(6):1506-1516.

14. Hayashi H, Inamura K, Aida K, Naoi S, Horikawa R, Nagasaka H, Takatani T, Fukushima T, Hattori A, Yabuki T, Horii I, Sugiyama Y: AP2 adaptor complex mediates bile salt export pump internalization and modulates its hepatocanalicular expression and transport function. Hepatology 2012, 55(6):1889-1900

15. Gonzales E, Grosse B, Cassio D, Davit-Spraul A, Fabre M, Jacquemin E: Successful mutation-specific chaperone therapy with 4-phenylbutyrate in a child with progressive familial intrahepatic cholestasis type 2 . J Hepatol 2012, 57(3):695-698.

16. Naoi S, Hayashi H, Inoue T, Tanikawa K, Igarashi K, Nagasaka H, Kage M, Takikawa H, Sugiyama Y, Inui A, Nagai T, Kusuhara H: Improved liver function and relieved pruritus after 4-phenylbutyrate therapy in a patient with progressive familial intrahepatic cholestasis type 2. $J$ Pediatr 2014, 164(5):1219-1227. e1213 
17. Davit-Spraul A, Fabre M, Branchereau S, Baussan C, Gonzales E, Stieger B, Bernard O, Jacquemin E: ATP8B1 and ABCB11 analysis in 62 children with normal gamma-glutamyl transferase progressive familial intrahepatic cholestasis (PFIC): phenotypic differences between PFIC1 and PFIC2 and natural history. Hepatology 2010, 51(5):1645-1655.

18. Pawlikowska L, Strautnieks S, Jankowska I, Czubkowski P, Emerick K, Antoniou A, Wanty C, Fischler B, Jacquemin E, Wali S, Blanchard S, Nielsen IM, Bourke B, McQuaid S, Lacaille F, Byrne JA, van Eerde AM, Kolho KL, Klomp L, Houwen R, Bacchetti P, Lobritto S, Hupertz V, McClean P, Mieli-Vergani G, Shneider B, Nemeth A, Sokal E, Freimer NB, Knisely AS, et al: Differences in presentation and progression between severe FIC1 and BSEP deficiencies. $J$ Hepatol 2010, 53(1):170-178.

19. Klomp LW, Vargas JC, van Mil SW, Pawlikowska L, Strautnieks SS, van Eijk MJ, Juijn JA, Pabón-Peña C, Smith LB, DeYoung JA, Byrne JA, Gombert J, van der Brugge G, Berger R, Jankowska I, Pawlowska J, Villa E, Knisely AS Thompson RJ, Freimer NB, Houwen RH, Bull LN: Characterization of mutations in ATP8B1 associated with hereditary cholestasis. Hepatology 2004, 40(1):27-38.

20. Strautnieks SS, Byrne JA, Pawlikowska L, Cebecauerová D, Rayner A, Dutton L, Meier Y, Antoniou A, Stieger B, Arnell H, Ozçay F, Al-Hussaini HF, Bassas $A F$, Verkade HJ, Fischler B, Németh A, Kotalová R, Shneider BL, CieleckaKuszyk J, McClean P, Whitington PF, Sokal E, Jirsa M, Wali SH, Jankowska I, Pawłowska J, Mieli-Vergani G, Knisely AS, Bull LN, Thompson RJ: Severe bile salt export pump deficiency: 82 different $A B C B 11$ mutations in 109 families. Gastroenterology 2008, 134(4):1203-1214.

21. Whitington PF, Whitington GL: Partial external diversion of bile for the treatment of intractable pruritus associated with intrahepatic cholestasis. Gastroenterology 1988, 95(1):130-136.

22. Nakamura K, Igarashi K, Ide K, Ohkawa R, Okubo S, Yokota H, Masuda A, Oshima N, Takeuchi T, Nangaku M, Okudaira S, Arai H, Ikeda H, Aoki J, Yatomi Y: Validation of an autotaxin enzyme immunoassay in human serum samples and its application to hypoalbuminemia differentiation. Clin Chim Acta 2008, 388(1-2):51-58.

23. Hayashi H, Takada T, Suzuki H, Akita H, Sugiyama Y: Two common PFIC2 mutations are associated with the impaired membrane trafficking of BSEP/ABCB11. Hepatology 2005, 41(4):916-924.

24. Kremer AE, Martens JJ, Kulik W, Ruëff F, Kuiper EM, van Buuren HR, van Erpecum KJ, Kondrackiene J, Prieto J, Rust C, Geenes VL, Williamson C, Moolenaar WH, Beuers U, Oude Elferink RP: Lysophosphatidic acid is a potential mediator of cholestatic pruritus. Gastroenterology 2010, 139(3):1008-1018. 1018 e1001.

25. Elman S, Hynan LS, Gabriel V, Mayo MJ: The 5-D itch scale: a new measure of pruritus. Br J Dermatol 2010, 162(3):587-593.

26. van der Velden LM, Stapelbroek JM, Krieger E, van den Berghe PV, Berger R, Verhulst PM, Holthuis JC, Houwen RH, Klomp LW, van de Graaf SF: Folding defects in P-type ATP 8B1 associated with hereditary cholestasis are ameliorated by 4-phenylbutyrate. Hepatology 2010, 51(1):286-296.

27. Aoki J, Inoue A, Okudaira S: Two pathways for lysophosphatidic acid production. Biochim Biophys Acta 2008, 1781(9):513-518.

28. Alemi F, Kwon E, Poole DP, Lieu T, Lyo V, Cattaruzza F, Cevikbas F, Steinhoff M, Nassini R, Materazzi S, Guerrero-Alba R, Valdez-Morales E, Cottrell GS, Schoonjans K, Geppetti P, Vanner SJ, Bunnett NW, Corvera CU: The TGR5 receptor mediates bile acid-induced itch and analgesia. J Clin Invest 2013, 123(4):1513-1530.

29. Kim SW, Hooker JM, Otto N, Win K, Muench L, Shea C, Carter P, King P, Reid AE, Volkow ND, Fowler JS: Whole-body pharmacokinetics of HDAC inhibitor drugs, butyric acid, valproic acid and 4-phenylbutyric acid measured with carbon-11 labeled analogs by PET. Nucl Med Biol 2013, 40(7):912-918.

30. van Mil SW, Klomp LW, Bull LN, Houwen RH: FIC1 disease: a spectrum of intrahepatic cholestatic disorders. Semin Liver Dis 2001, 21(4):535-544.

doi:10.1186/1750-1172-9-89

Cite this article as: Hasegawa et al: Intractable itch relieved by 4-phenylbutyrate therapy in patients with progressive familial intrahepatic cholestasis type 1. Orphanet Journal of Rare Diseases 2014 9:89.

\section{Submit your next manuscript to BioMed Central and take full advantage of:}

- Convenient online submission

- Thorough peer review

- No space constraints or color figure charges

- Immediate publication on acceptance

- Inclusion in PubMed, CAS, Scopus and Google Scholar

- Research which is freely available for redistribution

Submit your manuscript at www.biomedcentral.com/submit 